\title{
Immune Paralysis and the Spontaneous Remission of Cancer
}

\author{
BH Behzad Niakan*
}

P. O. Box 25817 Los Angeles CA. 90025 USA

*Corresponding author: BH Behzad Niakan, Pharm D, 10653 Lindbrook Drive, Los Angeles, CA 90024, USA, Tel: 310-9252720, E-mail: behzadniakan@yahoo.com

\section{Introduction}

Once a malignant growth grows past a certain size immune tolerance rather than immune sensitization occurs possibly due to the slow tumor growth. As the malignant grows larger some branches of the immune system (adoptive immunity) become protective of it, while other branches of the immune system oppose its growth. Overall, the protective branch of the immune system assures a continued malignant growth. This protective effect might be due to the immune system mistaking the malignant growth as self, due to immune tolerance. At rare instances such as a severe infection or an unusually large tumor mass the branches of the immune system protecting the malignant growth become overwhelmed and immune paralysis may occur. At this point a window is opened at which the malignant growth is not protected by some branches of the immune system. It is suggested that at this point a simple acute inflammation can be followed with a remission of the malignant disease.

An extensive and systemic review of the reports of the spontaneous remission of cancer seems to indicate in at least two areas the rejection of the malignant growth by the immune system could be associated with a temporary loss of the immune system protecting the malignant growth. (a) Severe infection; (b) An unusually large tumor burden.

Severe infection or sepsis can result in immune paralysis and there are some reports of the spontaneous remission of cancer after a severe infection $[1,2]$. Also, at times the tumor burden is unusually large, that is somehow the patients survived with a very large tumor burden, then immune system becomes overwhelmed in protecting the huge malignant growth and immune paralysis occurs. It is noted as the volume of the tumor increases, immune reactivity to the tumor deceases. There are some reports of the spontaneous remission of cancer both in human and mice after the tumor have grown to an unusually large size [3].

\section{Discussion}

(a) The immune system can be divided into two branches, Innate Immunity and adoptive immunity. Innate immunity can be divided into two parts, Cellular (platelets, NK Cells) and Humoral (Complement, Cytokines). NK cells as part of innate immunity may remove cancer cells. Innate immunity and adoptive immunity interact with one another and only one branch can be active at one time in response to a stimuli. Both, innate and adoptive immunity cannot be active at one time in response to the same stimuli.

(b) Innate immunity is the first line of defense. It recognizes foreign substance and dysfunctional or mal functional cells (Neoplasm). The information is passed from innate immunity to adaptive immunity to clear the body of unhealthy substances.

(c) Tumorigenes is occurs slowly and it does not induce sensitivity but tolerance and it escapes recognition by innate immunity. Once tolerance is developed adoptive immunity becomes protective of the malignant growth and at the time same suppresses innate immunity from initiating an immunological attack. Since, both innate immunity and adoptive immunity cannot be active simultaneously on the same case in response to the same stimuli.

(d) During an acute inflammatory response (Innate immunity) platelets activate NK cells in the removal of

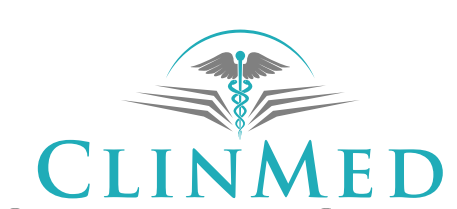

INTERNATIONAL LIBRARY

Citation: Niakan BHB (2017) Immune Paralysis and the Spontaneous Remission of Cancer. Int J Cancer Clin Res 4:087. doi.org/10.23937/2378-3419/1410087

Received: January 23, 2017; Accepted: November 14, 2017; Published: November 16, 2017

Copyright: (c) 2017 Niakan BHB. This is an open-access article distributed under the terms of the Creative Commons Attribution License, which permits unrestricted use, distribution, and reproduction in any medium, provided the original author and source are credited. 
malignant cells. Yet, during malignant growth platelets inhibit NK cells from removing malignant cells. Furthermore, immunoglobulins activate an immune response. Yet, during malignant growth immunoglobulins are ineffective in inducing an immune response against the malignant growth. Possibly it is the adaptive immunity that is reshaping the activity of innate immunity and humoral immunity.

As the tumor mass grows, immune activity against the malignant growth declines. This might be taken as "A decline in immunity activity against malignant growth corresponds with a decline in immune protection of malignant growth". In other words, at one point the immune system quits protecting the malignant growth and at this point the immune system is able to remove the malignant growth.

\section{Summary}

It is suggested that a short and temporary immune paralysis (suppression of adoptive immunity) followed with an acute inflammation could possibly be followed by the remission of the malignant disease. The immune system is mal functioning by protecting and rejecting the malignant growth. It is suggested that Once, the immune system quits protecting and/or rejecting the malignant growth, the immune system would readjust itself and the malignant growth is removed by the immune system.

\section{Suggested Experimental Treatment}

(1) Inducing an immune paralysis by an overload of antigen.

(2) Tuberculin skin test can be done. Once, delayed type hypersensitivity is completely diminished one might assume adaptive immunity is suppressed temporarily.

(3) Inducing an acute inflammation under the skin after immune paralysis is induced (As long as immune paralysis is present possibly adoptive immunity may not suppress innate immunity).

(4) As the host gradually clear itself of the antigen; repeatedly inducing an acute inflammation under the skin so that as soon as adoptive immunity becomes reactivated it would encounter the acute inflammatory response (prior to adoptive immunity suppressing innate immunity). This might shift the immune status to tumor rejection rather than tumor protection and rejection. Since, the acute inflammatory response might signal adoptive immunity that the malignant growth is foreign. The stronger the acute inflammation the more likely a remission.

(5) A low dose of corticosteroids (Dexamethasone, Etc.) might be helpful.

(6) A small and a low dose of Aspirin one hour before inducing an acute inflammatory response might enhance the effect. Caution should be exercised due to the possibility of life threatening internal bleeding. Aspirin may be given if the patient does not have a condition that would preclude the use of Aspirin. Aspirin has a long half-life and a second dose may be given in two days if there is no drug interaction or the patient does not have a condition that would preclude the use of aspirin.

(7) Injecting plasma rich platelet at the site of the acute inflammation might have an enhancing effect.

(8) Temporarily suppressing cellular immunity might enhance the effect. Drugs used to suppress graft rejection might be used. Caution should be exercised when using these drugs for their potential life threatening effect.

\section{Caution}

Medical Supervision Required. A patient condition may preclude use of non-live vaccine. There is no proof that the suggested experimental treatment would work. It is based on a hypothesis suggesting an immunological mechanism for the spontaneous remission of cancer after acute infections. Therefore, suggesting an experimental treatment for cancer patients.

\section{Disclaimer}

No content on this site regardless of date should ever be used as a substitute for direct medical advice from your doctor or other qualified clinicians. Author requesting anyone with any information regarding non live vaccines and cancer to send an email to behzad.niakan@yahoo.com

\section{References}

1. Køstner AH, Johansen RF, Schmidt H, Mølle I (2013) Regression in cancer following fever and acute infection. Acta Oncol 52: 455-457.

2. Jonathan I Huz, Marcovalerio Melis, Umut Sarpel (2012) Spontaneous regression of hepatocellular carcinoma most often associated with tumor hypoxia or a systemic inflammatory response. HPB (Oxford) 14: 500-505.

3. Behzad Niakan (1993) Steady tumor growth and the spontaneous disappearance of cancer. J Natl Cancer Inst 85: 68-69. 